\title{
Oxide-Trapped Charges Induced by Electrostatic Discharge Impulse Stress
}

\author{
Jen-Chou Tseng and Jenn-Gwo Hwu
}

\begin{abstract}
The characteristics of oxide-trapped charges $Q_{\text {ot }}$ induced by electrostatic discharge high-field current impulse stress, i.e., transmission line pulsing (TLP), were studied. It was observed that for a 3.2-nm-thin oxide, the centroid evolution and the critical density of positive oxide-trapped charges $Q_{\text {ot }}^{+}$to trigger oxide breakdown are about the same between dc and TLP impulse stresses. These results are consistent with the existing models of stress-induced trapping charges and hole-induced oxide breakdown. However, different behaviors of $Q_{\mathrm{ot}}$ and centroid were found for 14-nm-thick oxides subjected to different stress tests. TLP impulse stress generates far less amount of negative oxidetrapped charges $Q_{\text {ot }}^{-}$than dc stress, and the positive oxide-trapped charges finally dominate over the negative oxide-trapped charges. This impulse stress imposes a high density and transient current on the oxide, which induces traps at the tunneling distance locally. The hotter injected electrons generate more efficient hole trappings to provoke breakdown with lower density of oxide-trapped charges in comparison with de stress test.
\end{abstract}

Index Terms-Electrostatic discharge (ESD), gate dielectrics, MOS devices, oxide-trapped charges, semiconductor device reliability, transmission line pulsing (TLP).

\section{INTRODUCTION}

A S SCALING of integrated circuit technology continues, it is hard to ensure the robustness of gate oxides in an electrostatic discharge (ESD) event. This is particularly important for input and input-output circuits in high-speed and high-performance applications, where transistor gates may be directly connected to an external pin without protection of resistor or adopted as decoupling capacitors between powers, which exposes the thin gate oxides to ESD stress. The gate oxides may breakdown catastrophically or suffer latent damage to impact the long-term reliability. The reliability of gate oxides has been extensively studied, including the effects of stress-induced oxide-trapped charges $Q_{\text {ot }}$ and the electrical breakdown mechanism. Most of the studies are performed under constant voltage stress such as time-dependent dielec-

Manuscript received October 24, 2006; revised March 9, 2007. This work was supported in part by the National Science Council of Taiwan, R.O.C., under Contract NSC 95-2221-E-002-375. The review of this paper was arranged by Editor J. Suehle.

J.-C. Tseng is with the Department of Electrical Engineering and Graduate Institute of Electronics Engineering, National Taiwan University, Taipei 106, Taiwan, R.O.C., and also with Winbond Electronics Corporation, Hsinchu 300, Taiwan, R.O.C

J.-G. Hwu is with the Department of Electrical Engineering/Graduate Institute of Electronics Engineering, National Taiwan University, Taipei 106, Taiwan, R.O.C. (e-mail: hwu@cc.ee.ntu.edu.tw).

Color versions of one or more of the figures in this paper are available online at http://ieeexplore.iee.org.

Digital Object Identifier 10.1109/TED.2007.899429 tric breakdown and stress-induced leakage current. According to the hole-trapping oxide breakdown model, the electrical breakdown of thin silicon oxide is a two-step process [1]-[5]. The first is the buildup stage, where the positive oxide-trapped charges $Q_{\mathrm{ot}}^{+}$resulted from hole generation, which is created by impact ionization in the oxide, and led to local high-field and high-current-density regions. When the localized highfield/high-current regions reach a critical value, the brief second stage begins, where high current densities cause thermal runaway and provoke catastrophic breakdown. Additionally, highfield stresses induce oxide-trapped charges in the oxide film that can result in threshold voltage shifts, excess leakage currents, and degradation of oxide breakdown [6]-[12]. Previous studies have successively determined most trap parameters by various techniques, and the distributions of oxide-trapped charges were measured and discussed to analyze trapping mechanisms [13]-[17]. However, only a few studies were related to the effect of impulse stress with very high electric field and very short impulse duration that are presented during an ESD event [18]-[25]. As reported, the mechanism of gate-oxide degradation remains unchanged over the range of $10^{2}-10^{-8} \mathrm{~s}$; the time to breakdown is expected to exhibit an approximately $1 / E$ dependence. The oxide breakdown limits between $\mathrm{dc}$ and short pulses were compared, two decades of current difference are noticed, and breakdown voltage was increased by a factor of 1.3-1.6. Nevertheless, a definitive picture of the formations of oxide-trapped charges induced by ESDlike impulse is still missing. For this reason, this paper extends the aforementioned studies by looking at the effects of high-field current impulse on the formation of stress-induced oxide-trapped charges and breakdown characteristics of MOS capacitors.

Transmission line pulsing (TLP) curve tracer test systems can provide high-field current impulses and have been widely applied to examine the electrical characteristics of semiconductor device during very short impulse durations and also during the emulation of ESD [26]. A TLP tester with 100-ns pulsewidth and 10-ns rise/fall time can simulate and correlate the performance of human body model ESD stress on semiconductor devices. This paper explores the density and centroid of stressinduced oxide-trapped charges both for long durations such as $\mathrm{dc}$ and for very short durations below the microsecond level such as TLP impulse. Different formations of oxide-trapped charges induced by different manners of current injection were examined. The characteristics of oxide-trapped charges and breakdown limit were analyzed for 3.2-nm-thin and 14-nmthick oxides. 


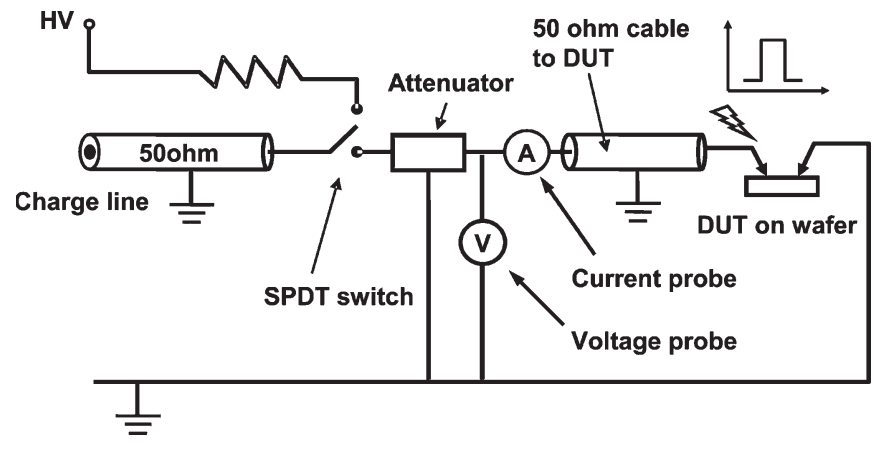

Fig. 1. TLP setup configuration. Square waveforms with 100 -ns pulsewidth and 10-ns rise/fall time were applied to a device, whereas the voltage across and the current through it were measured.

\section{EXPERIMENTAL SETUP}

Experiments were performed on MOS capacitors with 3.2-nm-thin and 14-nm-thick gate oxides, which are fabricated by a standard $0.18-\mu \mathrm{m}$ dual-gate-oxide complementary MOS technology. Features of this process included conventional shallow trench isolation, twin well, nonsilicide source/drain, dualpoly gate $\left(\mathrm{n}^{+} / \mathrm{p}^{+}\right)$with tungsten silicide cap, and tungsten plugged contact. The MOS capacitors are fingers in shape with p-type substrates. For 3.2-nm samples, the width and length are 0.16 and $150 \mu \mathrm{m}$, respectively. For 14-nm samples, the width and length are 0.6 and $200 \mu \mathrm{m}$, respectively. The current-voltage $(I-V)$ characteristics and dc constant current stress were performed by HP 4156C. TLP characteristics were measured by a commercial Barth 4002 pulse curve tracer system. This TLP system applied the square waveform with 100-ns pulsewidth and 10-ns rise/fall time to a device while measuring the voltage across and the current through it. Fig. 1 illustrates the TLP setup configuration [26]. As the figure shows, the transmission line is a $50-\Omega$ coaxial cable charged by the $\mathrm{dc}$ high-voltage generator. The discharge voltage was applied and converted into a current pulse by a resistor. The current pulse with square waveform rises with the voltage provided from the dc high-voltage generator. Constant current pulses were then forced into the device, enabling the behavior of devices under high-current conditions to be studied.

To quantify the amount of density and centroid of the oxidetrapped charges in bulk oxide, a bidirectional $I-V$ measurement was adopted [13]. This technique involves measuring the voltage shifts on the $I-V$ characteristics (both positive and negative biases) of the oxide film after each stress of an accumulated stress sequence. Trapped charge density $Q_{\text {ot }}$ and its centroid $\bar{x}$ can then be calculated by the following equations [13]:

$$
\begin{aligned}
\Delta V g^{-} & =\frac{1}{\varepsilon}(L-\bar{x}) \cdot Q_{\mathrm{ot}} \\
\Delta V g^{+} & =-\frac{\bar{x}}{\varepsilon} Q_{\mathrm{ot}} \\
Q_{\mathrm{ot}} & =(\varepsilon / L) \cdot\left(\Delta V g^{-}-\Delta V g^{+}\right) \\
\bar{x} / L & =\left[1-\left(\Delta V g^{-}-\Delta V g^{+}\right)\right]^{-1}
\end{aligned}
$$

where $\Delta V g^{-}$and $\Delta V g^{+}$are parallel shifts along the voltage axis of the positive and negative $I-V$ plots, respectively, that

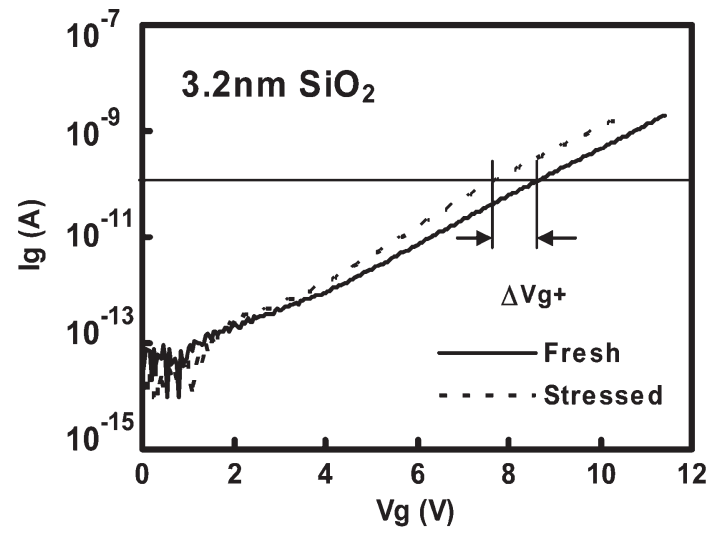

(a)

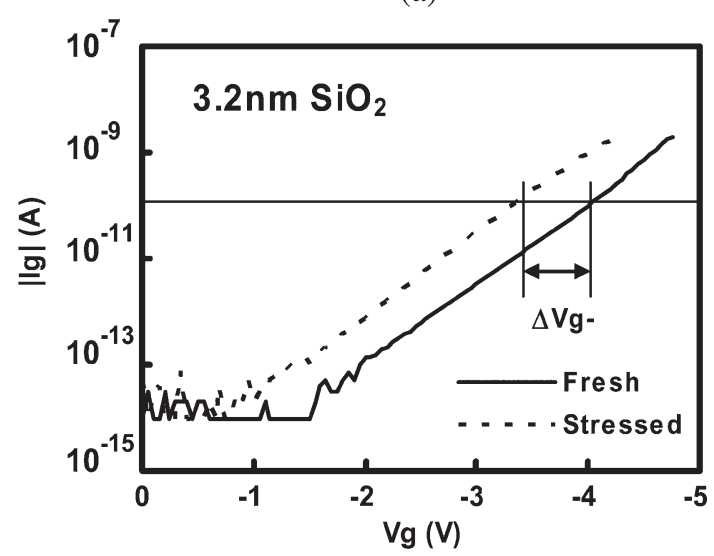

(b)

Fig. 2. Typical $I-V$ characteristics for a 3.2-nm gate-oxide MOS capacitor before and after stress. The superscripts (a) + and (b) - refer to positive and negative gate polarities, respectively.

are measured between the prestress and poststress $I-V$ plots. $Q_{\text {ot }}$ is the total oxide-trapped charges over the oxide thickness $L . \bar{x}$ is the centroid of the trapped charges, and $\varepsilon$ is the insulator low-frequency dielectric constant. The superscripts + and - refer to positive and negative gate polarities, respectively. Typical $I-V$ characteristics for 3.2-nm gate-oxide MOS capacitors before and after stresses are shown in Fig. 2. To minimize any undesirable trapping of oxide charges that might occur during $I-V$ measurement, the current densities in these measurements are kept at least two orders of magnitude lower than the stressing current. In our calculation, these values were evaluated at a fixed current level of $100 \mathrm{pA}$. The gate-oxide interface is used as the origin of coordinates.

Experimental procedures are described as follows: First, the bidirectional $I-V$ characteristics were measured for initial parameters check. Then, MOS capacitors were subjected to current stress using dc and TLP sources separately. After a known time interval of dc stress and one impulse of TLP stress, the stressing was interrupted, and bidirectional $I-V$ plots were remeasured. This procedure is repeated until the breakdowns.

\section{RESULTS AND DISCUSSIONS}

This section presents the stress-induced oxide-trapped charges and the breakdown of 3.2- and 14-nm gate oxides. The discussions comprised two parts. First, the density and 


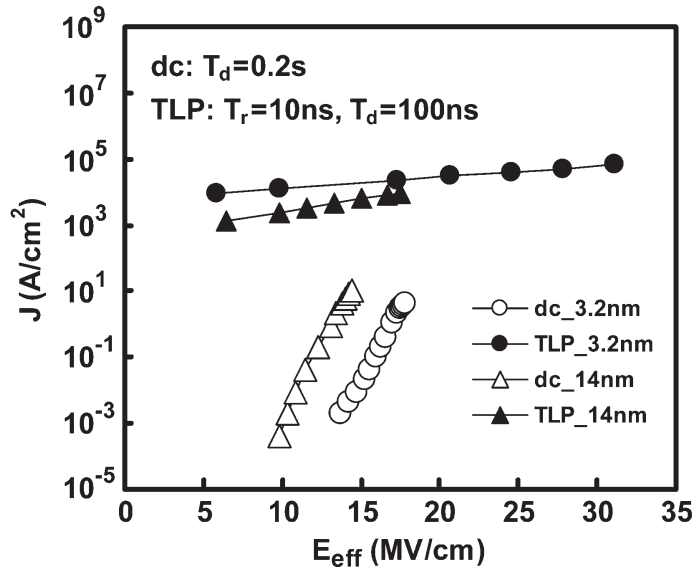

Fig. 3. $J-E_{\text {eff }}$ characteristics of dc and TLP impulse measurements on MOS capacitors for both 3.2-nm-thin and 14-nm-thick gate oxides.

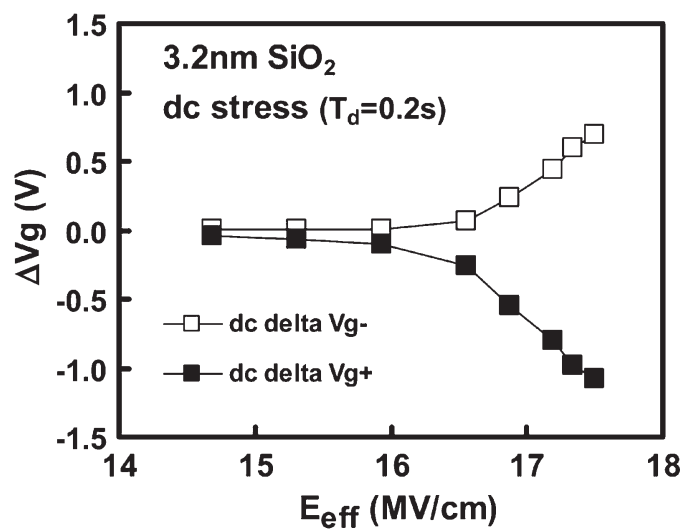

(a)

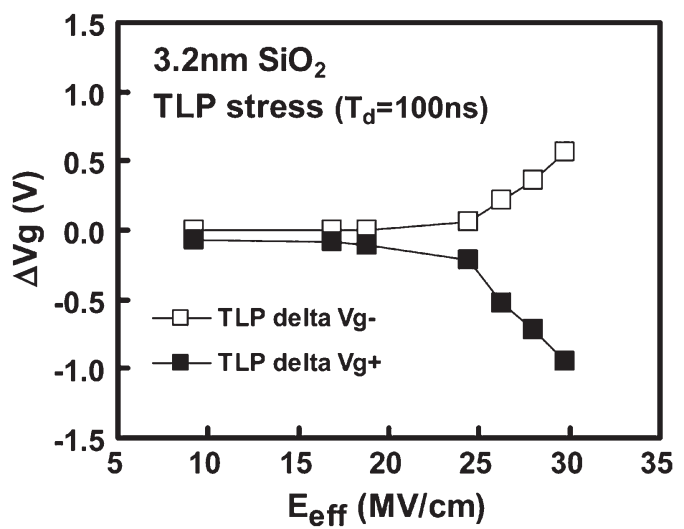

(b)

Fig. 4. Gate voltage shifts versus electric field for 3.2-nm MOS capacitors subjected to (a) dc and (b) TLP impulse stress tests.

centroid of oxide-trapped charges induced by dc and TLP impulse stresses were investigated. Then, the corresponding mechanisms of trapped charge generation and breakdown were analyzed, and possible models were proposed for the two stress types.

\section{A. 3.2-nm-Thin Gate Oxide Under DC and TLP Impulse Stressing}

High-field electrical stress to the thin oxide in the MOS devices causes Fowler-Nordheim electron tunneling. Fig. 3

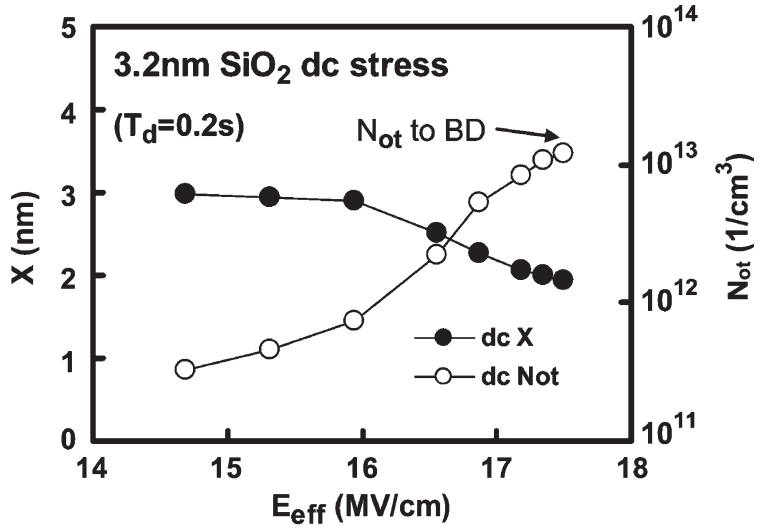

(a)

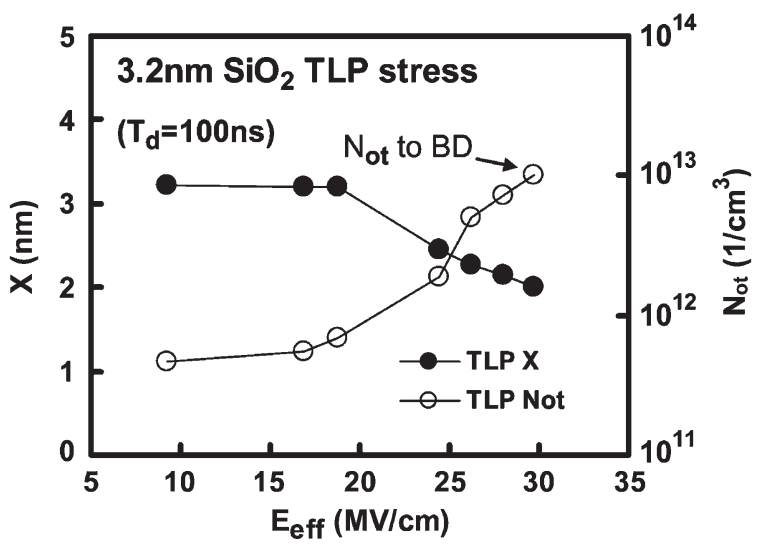

(b)

Fig. 5. Extracted density of oxide-trapped charges $N_{\text {ot }}$ and centroid in the oxide against electric field for 3.2-nm NMOS capacitors subjected to (a) dc and (b) TLP impulse stress tests.

plots the $J-E_{\text {eff }}$ characteristics of dc and TLP impulse measurements on MOS capacitors for both 3.2-nm-thin and 14-nmthick gate oxides. In the accumulation region of p-type substrate during stress, the voltage drop across $\mathrm{Si}$ substrate is negligible. Therefore, $E_{\text {eff }}$ is defined as the ratio of applied voltage to oxide thickness. Significantly, at least two decades of current difference are noticed, and breakdown voltage is increased by a factor of 1.2-1.7 when we compare the dc and TLP impulse stress tests. These data are similar to the results of previous studies [20]-[23]. Fig. 4(a) and (b) shows the gate voltage shifts versus electric field for 3.2-nm gate-oxide MOS capacitors subjected to dc and TLP impulse stress tests, respectively. Both stress tests exhibited the same trend of voltage shifts that they continue increasing with the rising electric field. However, the electric field of TLP impulse stress is about 1.7 times as large as that of dc stress to achieve the same voltage shift. Fig. 5(a) and (b) shows the plots of the extracted density of oxide-trapped charges $N_{\text {ot }}$ and centroid in the oxide against electric field for 3.2-nm gate-oxide MOS capacitors subjected to dc and TLP impulse stress tests, respectively. Clearly, positive oxide-trapped charges (holes) were indicated, and their density of breakdown limits for both stress tests were of about the same level. Besides, similar trend of centroid reveals that the hole generation is near anode and then drifted toward bulk due to the applied electric field. These results match the oxide breakdown model of hole trapping; that is, breakdown occurs 


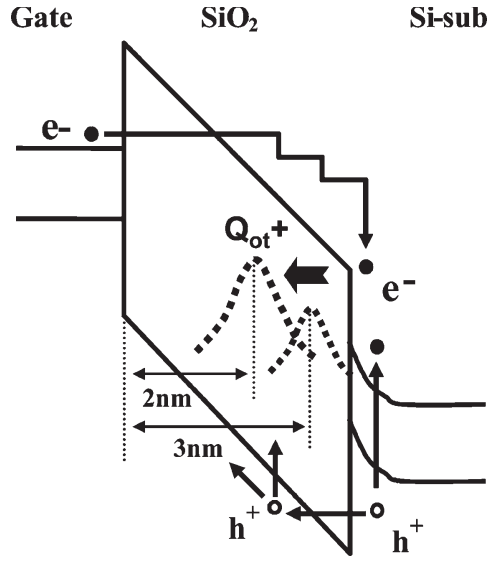

Fig. 6. Schematic diagram of the generation of oxide-trapped charges for 3.2-nm MOS capacitors subjected to dc and TLP impulse stress tests. Hot electrons are generated by band-to-band impact ionization, and part holes were trapped near the $\mathrm{SiO}_{2} /$ substrate interface. The hole generation is initially near the anode and then drifts toward bulk due to the applied electric field.

when a critical value of the density of trapped holes is reached for both dc and TLP impulse stress tests.

Fig. 6 illustrates the schematic diagram of the generation of $Q_{\text {ot }}$ for 3.2-nm gate-oxide NMOS capacitors subjected to dc and TLP impulse stress tests. The hot electrons are generated by band-to-band impact ionization, and part holes were trapped near the $\mathrm{SiO}_{2} /$ substrate interface. The hole generation is initially near the anode and then drifts toward bulk due to the applied electric field.

\section{B. 14-nm Gate Oxide Under DC and TLP Impulse Stresses}

According to earlier publications, the stress-induced neutral oxide traps are located slightly to the cathode side due to the electron injection damages, and the generated neutral oxide traps distribute almost uniformly in the oxide [7], [11], [12]. These neutral traps catch electrons and become negatively charged as the applied electric field rises. The centroid of the negative charges is at the middle of the oxide. Additionally, although electron trapping occurs throughout the stressing experiment, it is not responsible for oxide breakdown [9]. The hole trapping in localized areas at the cathode is responsible for oxide breakdown subjected to high current stress. Localized hole trapping enhances the cathode field and increases the local current density, hence further accelerating the local hole trapping. This process eventually causes the local current density to reach a critical value and cause a destructive thermal breakdown [1]-[3].

Fig. 7(a) and (b) shows the gate voltage shifts versus electric field for 14-nm gate-oxide MOS capacitors subjected to dc and TLP impulse stress tests, respectively. DC stress results showed increasing negative voltage shifts and turnaround positive voltage shifts. However, TLP impulse stress induced far less voltage shifts than dc stress and revealed rebound-like behaviors that the voltage shifts finally changed their polarities. Besides, to trigger oxide breakdown, the electric field of TLP impulse stress is about 1.2 times as large as that of dc stress. Fig. 8(a) and (b) shows the plots of the extracted $N_{\text {ot }}$ and centroid in the oxide versus electric field for 14-nm

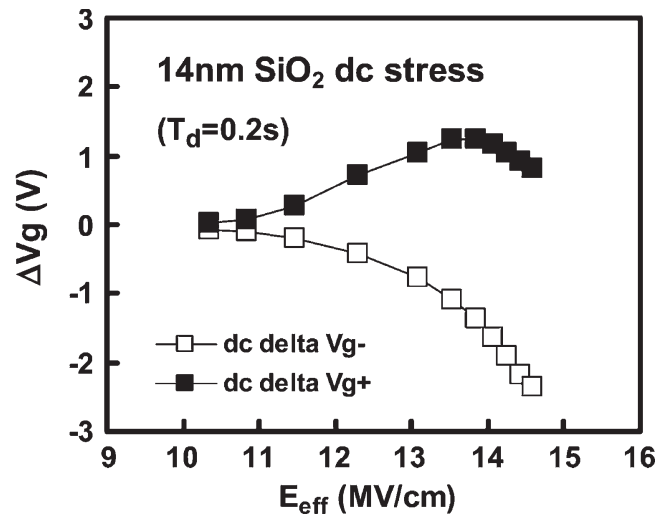

(a)

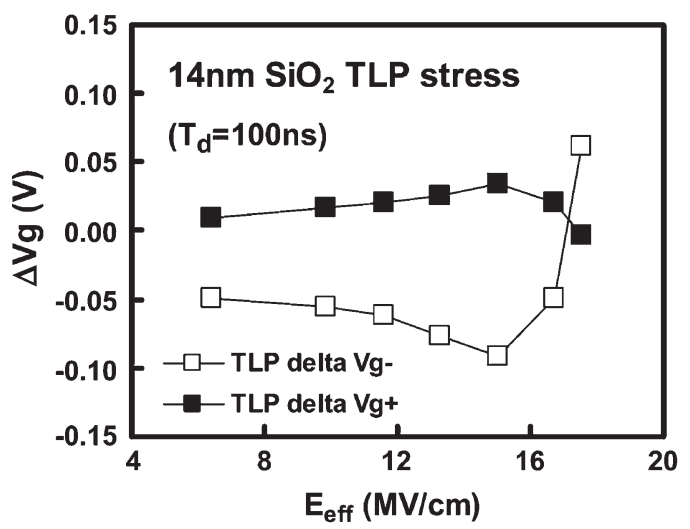

(b)

Fig. 7. Gate voltage shifts versus electric field for 14-nm MOS capacitors subjected to (a) dc and (b) TLP impulse stress tests.

gate-oxide MOS capacitors subjected to dc and TLP impulse stress tests, respectively. Turnaround of the centroid is observed for both stress tests. The negative oxide-trapped charges were first generated at the cathode side, and they drifted toward the anode by the applied electric field afterward. Then, mass positive oxide-trapped charges were generated at the cathode side and eventually pulled back the centroid to the cathode side. For dc stress test, a large amount of negative oxide-trapped charges were generated due to the abundant stress-induced traps, and the peak of the centroid was approximately located at the middle of the oxide. However, for TLP impulse stress test, less negative oxide-trapped charges were generated, the centroid position was around the tunneling distance of injected electrons (3 $\mathrm{nm}[6])$, and the polarity of final oxide-trapped charges changed from negative to positive. Fig. 8(c) shows the plot of the generation rate of $\Delta N_{\text {ot }} / \Delta E_{\text {eff }}$ versus $E_{\text {eff }}$. The $N_{\text {ot }}$ generation rates were extracted and showed quite different behaviors. Generation rates of dc stress were much larger than those of TLP impulse stress since dc injection is slow and uniform as compared with ESD injection [27]. Under dc stress test, the negative trapped charges were fast generated with the increasing electric field. As the applied field continues rising, the $N_{\text {ot }}$ generation rate is observed to decay. One root cause is that the traps are filled without significant tunneling out of traps. Another is attributed to the generation of positive trapped charges that provoke the breakdown. On the other hand, the TLP impulse imposes a very high field and transient current 


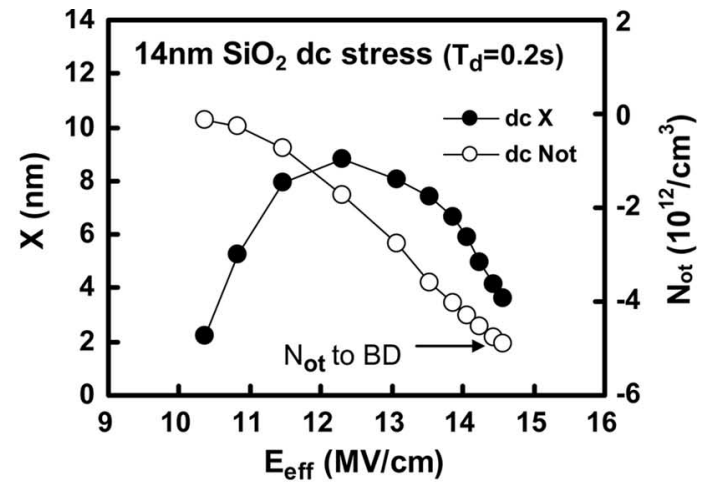

(a)

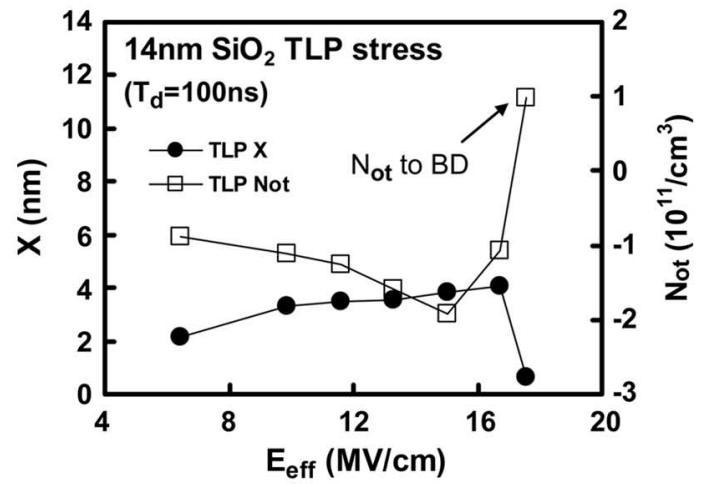

(b)

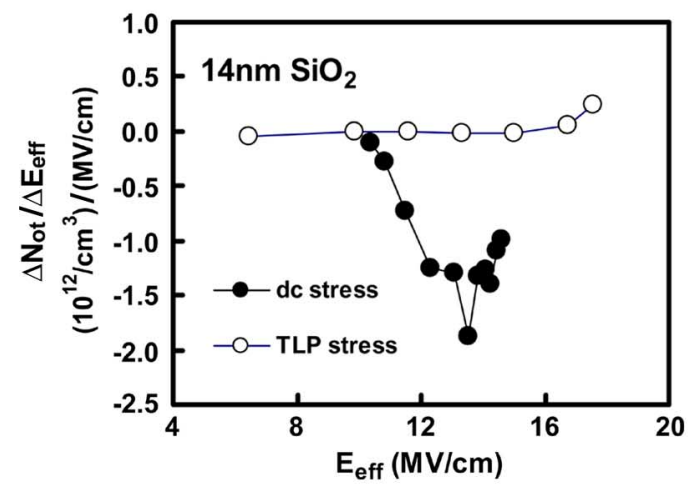

(c)

Fig. 8. Plots of the extracted $N_{\text {ot }}$ and centroid in the oxide versus electric field for 14-nm MOS capacitors subjected to (a) dc and (b) TLP impulse stress tests. (c) Generation rate of $\Delta N_{\text {ot }} / \Delta E_{\text {eff }}$ versus $E_{\text {eff }}$.

on the oxide. As reported in previous publications, device is prone to current filament and localization under high-current conditions [28]. Therefore, a nonuniform current path results in lower $N_{\text {ot }}$ generation and less voltage shifts under TLP impulse stressing.

Fig. 9 illustrates the schematic diagram of the generation of $Q_{\text {ot }}$. For dc stress test, mass traps are generated, and trapassisted tunneling is resulted. As the applied electric field is raised further, the negative oxide-trapped charges are formed uniformly in the oxide. Additionally, positive oxide-trapped charges are generated near the cathode side by the trap-assisted impact ionization and finally lead to oxide breakdown. On the other hand, TLP impulse stress generates traps locally at the tunneling distance due to injection damage and induces higher electric field. Hotter injected electrons cause band-to-

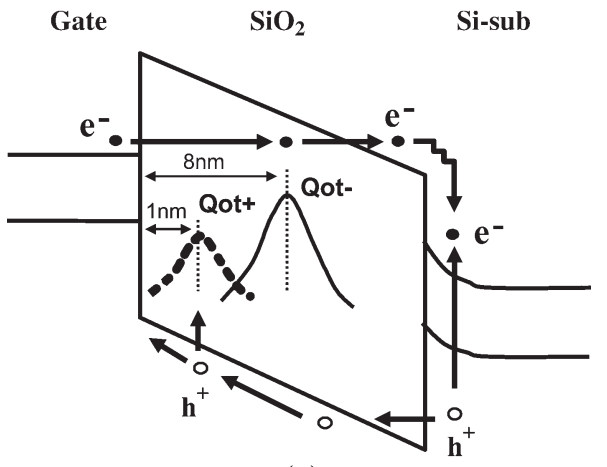

(a)

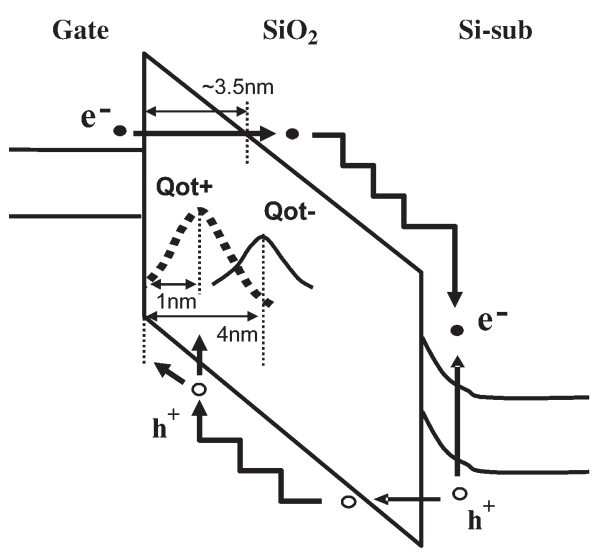

(b)

Fig. 9. Schematic diagrams of the generations of oxide-trapped charges for 14-nm MOS capacitors subjected to (a) dc and (b) TLP impulse stress tests.

band impact ionization and therefore generate more efficient hole trappings than dc stress test.

\section{CONCLUSION}

The characteristics of oxide-trapped charges in oxide subjected to dc and ESD high-field current impulse (TLP impulse) stress have been obtained and analyzed. Both thin and thick oxides were examined. For thinner oxide $(3.2 \mathrm{~nm})$, the centroid moves from substrate interface toward gate interface (cathode interface) for both stress types. The critical density and polarity of oxide-trapped charges to provoke breakdown are also about the same for both cases. However, for the 14-nm-thick oxide case, dc stress induces mass oxide-trapped charges by trapassisted impact ionization, whereas TLP impulse stress generates far less negative oxide-trapped charges than dc stress. It was suggested that hotter injected electrons cause band-toband impact ionization and therefore induce more efficient hole trappings to provoke breakdown with lower density of oxidetrapped charges as compared with dc stress test. The roles of oxide-trapped charges are essentially different between dc and TLP stresses and cause different effects on oxide breakdowns.

\section{REFERENCES}

[1] S. Holland, I. C. Chen, T. P. Ma, and C. Hu, "On physical models for gate oxide breakdown," IEEE Electron Device Lett., vol. EDL-5, no. 8, pp. 302-305, Aug. 1984.

[2] I.-H. Chen, S. E. Holland, and C. Hu, "Electrical breakdown in thin gate tunneling oxides," IEEE Trans. Electron Devices, vol. ED-32, no. 2, pp. 413-416, Feb. 1985. 
[3] I. C. Chen, S. Holland, and C. Hu, "Oxide breakdown dependence on thickness and hole current-enhanced reliability of ultra thin oxides," in IEDM Tech. Dig., 1986, vol. 32, pp. 660-663.

[4] E. Vincent, "(Ultra) Thin oxide breakdown(s), An overview," in Proc. IEEE Integr. Reliab. Workshop Final Report, 1999, pp. 158-166.

[5] D. J. Dumin, S. K. Mopuri, S. Vanchinathan, R. S. Scott, R. Subramoniam, and T. G. Lewis, "High field related thin oxide wearout and breakdown," IEEE Trans. Electron Devices, vol. 42, no. 4, pp. 760-772, Apr. 1995.

[6] M. S. Liang, C. Chang, Y. T. Yeow, C. Hu, and R. W. Brodersen, "MOSFET degradation due to stressing of thin oxide," IEEE Trans. Electron Devices, vol. ED-31, no. 9, pp. 1238-1244, Sep. 1984.

[7] C. S. Jeng, T. R. Ranganath, C. H. Huang, H. S. Jones, and T. T. L. Chang, "High-field generation of electron traps and charge trapping in ultra-thin $\mathrm{SiO}_{2}, "$ in IEDM Tech. Dig., 1981, vol. 27, pp. 388-391.

[8] M. S. Liang and C. Hu, "Electron trapping in very thin thermal silicon dioxides," in IEDM Tech. Dig., 1981, vol. 27, pp. 396-399.

[9] M. S. Liang, S. Haddad, W. Cox, and S. Cagnina, "Degradation of very thin gate oxide MOS devices under dynamic high field/current stress," in IEDM Tech. Dig., 1986, pp. 394-398.

[10] R. Moazzami and C. Hu, "Stress-induced current in thin silicon dioxide films," in IEDM Tech. Dig., Dec. 13-16, 1992, pp. 139-142.

[11] M. Kimura and H. Koyama, "Stress-induced low-level leakage mechanism in ultrathin silicon dioxide films caused by neutral oxide trap generation," in Proc. IEEE Int. Reliab. Phys. Symp., Apr. 1994, pp. 167-172.

[12] R. S. Scott, N. A. Dumin, T. W. Hughes, D. J. Dumin, and B. T. Moore, "Properties of high-voltage stress generated traps in thin silicon oxide," IEEE Trans. Electron Devices, vol. 43, no. 7, pp. 1133-1143, Jul. 1996.

[13] D. J. Dimaria, "Determination of insulator bulk trapped charge densities and centroids from photocurrent-voltage characteristics of MOS structures," J. Appl. Phys., vol. 47, no. 9, pp. 4073-4077, Sep. 1976.

[14] R. Kies, T. Egilsson, G. Ghibaudo, and G. Pananakakis, "A method for the assessment of oxide charge density and centroid in metal-oxide-semiconductor structures after uniform gate stress," Appl. Phys. Lett., vol. 26, no. 68, p. 3790, Jun. 1996.

[15] Y. H. Lin, C. L. Lee, and T. F. Lei, "Monitoring trapped charge generation for gate oxide under stress," IEEE Trans. Electron Devices, vol. 44, no. 9, pp. 1441-1446, Sep. 1997.

[16] R. Rodriguez, M. Nafria, J. Sune, and X. Aymerich, "Trapped charge distributions in thin $(10 \mathrm{~nm}) \mathrm{SiO}_{2}$ films subjected to static and dynamic stresses," IEEE Trans. Electron Devices, vol. 45, no. 4, pp. 881-888, Apr. 1998.

[17] K. Kobayashi, A. Teramoto, and H. Miyoshi, "Origin of positive charge generated in thin $\mathrm{SiO}_{2}$ films during high-field electrical stress," IEEE Trans. Electron Devices, vol. 46, no. 5, pp. 947-953, May 1999.

[18] M. A. Bridgwood and R. H. Kelley, "Modeling the effects of narrow impulsive overstress on capacitive test structures," in Proc. EOS/ESD Symp., 1985, pp. 84-91.

[19] E. A. Amerasekera and D. S. Campbell, "ESD pulse and continuous voltage breakdown in MOS capacitor structure," in Proc. EOS/ESD Symp., 1986, pp. 208-213.

[20] Y. Fong and C. Hu, "The effects of high electric field transients on thin gate oxide MOSFETS," in Proc. EOS/ESD Symp., 1987, pp. 252-257.

[21] W. K. Chim and G. L. Teh, "Neutral electron trap generation and hole trapping in thin oxides under electrostatic discharge stress," Jpn. J. Appl. Phys., vol. 37, no. 4A, pp. 1671-1673, 1998.

[22] H. Gieser and M. Haunschild, "Very-fast transmission line pulsing of integrated structures and the charged device model," in Proc. EOS/ESD Symp., 1996, pp. 85-94.

[23] C. Leroux, P. Andreucci, and G. Reimbold, "Analysis of oxide breakdown mechanism occurring during ESD pulse," in Proc. IEEE Int. Reliab. Phys. Symp., 2000, pp. 276-282.

[24] J. Wu and E. Rosenbaum, "Gate oxide reliability under ESD-like pulse stress," IEEE Trans. Electron Devices, vol. 51, no. 9, pp. 1528-1532, Sep. 2004.
[25] B. E. Weir, C.-C. Leung, P. J. Silverman, and M. A. Alam, "Gate dielectric breakdown: A focus on ESD protection," in Proc. Int. Reliab. Phys. Symp., 2004, pp. 399-404.

[26] J. Barth, J. Richner, K. Verhaege, and L. G. Henry, "TLP calibration, correlation, standards, and new techniques," in Proc. EOS/ESD Symp., 2000, pp. 85-96.

[27] S. Aur, A. Chatterjee, and T. Polgreen, "Hot-electron reliability and ESD latent damage," IEEE Trans. Electron Devices, vol. 35, no. 12, pp. 21892193, Dec. 1988

[28] A. Amerasekera and C. Duvvury, ESD in Silicon Integrated Circuits, 2nd ed. Hoboken, NJ: Wiley, 2002

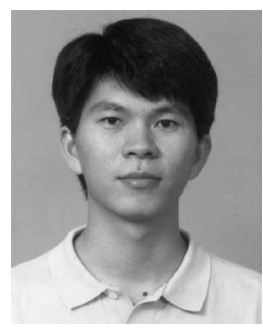

Jen-Chou Tseng received the B.S. degree from National Chiao-Tung University, Hsinchu, Taiwan, R.O.C., in 1992 and the M.S. degree from National Tsing-Hua University, Hsinchu, in 2000. He is currently working toward the Ph.D. degree at National Taiwan University, Taipei, Taiwan

From 1994 to 1998, he was with Holtek Semiconductor Corporation, Hsinchu, as a Section Head in process integration engineering, where he was responsible for yield enhancement. Since 2000, he has been with Winbond Electronics Corporation, Hsinchu, as a Section Manager in device engineering, where he is responsible for electrostatic discharge (ESD)/latch-up protection design. His current research interest is in ESD characterization of gate dielectrics.

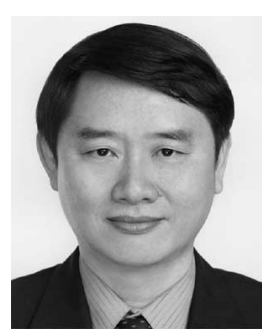

Jenn-Gwo Hwu was born in Tainan, Taiwan, R.O.C., on August 29, 1955. He received the B.S. degree in electronic engineering from National Chiao-Tung University, Hsinchu, Taiwan, in 1977 and the M.S. and Ph.D. degrees in electrical engineering from National Taiwan University, Taipei, Taiwan, in 1979 and 1985, respectively.

He joined the faculty of National Taiwan University in 1981. From 1997 to 1998 , he was the Vice Chairman of the Department of Electrical Engineering, National Taiwan University. From February 2004 to January 2006, he was invited as the Dean of the College of Electrical Engineering and Computer Science, National United University, Miaoli, Taiwan, R.O.C. From December 2005, he was invited as the Coordinator of Micro-Electronics Engineering Program, Department of Engineering and Applied Sciences, National Science Council, Taipei. On August 2006, he was appointed as the Distinguished Professor of the National Taiwan University $\mathrm{He}$ is currently a Professor in the Department of Electrical Engineering and Graduate Institute of Electronics Engineering, National Taiwan University. His research interest is mainly on ultrathin gate oxide and its related $\mathrm{Si}$ MOS devices. He has experience in teaching the courses of circuits, electronics, solidstate electronics, semiconductor engineering, MOS capacitor devices, radiation effects on MOS system, and special topic on oxide reliability.

Prof. Hwu was qualified to be a licensed Professional Technique Experts on Electrical and Electronics Engineering, Taiwan, in 1978 and 1980. He was honored as the owner of Outstanding Teaching Award by the Ministry of Education in 1991 and by the National Taiwan University in 1987 and 2003. He was also the owner of Excellent Teaching Award by the College of Engineering, National Taiwan University, in 1988 to 1991, and 1993 and by the National Taiwan University in 1999, 2000, and 2002. He was the recipient of the Jan Ten-You Paper Award by the Chinese Institute of Engineering, Taiwan, in 1999 and of the Scientific Paper Award by the Far Eastern Y. Z. Hsu Science and Technology Memory Foundation, Taiwan, in 2005. 